\title{
In situ TEM Investigation of the Electroplasticity Phenomenon in Metals
}

Xiaoqing $\mathrm{Li}^{1,2^{*}}$ and Andrew M. Minor ${ }^{1,2}$.

1. University of California Berkeley, Department of Materials Sciences and Engineering, Berkeley, USA.

2. National Center for Electron Microscopy (NCEM) at Molecular Foundry, Lawrence Berkeley National Laboratory (LBNL, LBL), Berkeley, CA, USA.

* Xiaoqing Li: li_xiaoqing@berkeley.edu

Electroplasticity (EP) is a phenomenon in which applied pulsed electric fields during deformation result in increased formability in metals. This phenomenon has been studied since the 1950's [1] and researchers have proposed two possible origins of this nearly-universal effect: (1) localized Joule heating at lattice defects and (2) the electron wind effect leading to vacancy migration [2]. However, there is no clear evidence of the true origin of this behavior at the level of individual defects [3]. In this work, in situ Transmission Electron Microscope (TEM) electromechanical tests were performed in order to correlate direct observations of dislocation motion with both mechanical data and applied electrical pulses in an effort to provide clarity on the true origin of this interesting phenomenon.

In this study we have systematically studied the way electrical fields alter the structure and mobility of dislocations in metals such as $\mathrm{Al}, \mathrm{Ti}$ and $\mathrm{Ni}$. The controlled electromechanical experiments were performed during in situ testing in a JEOL 3010 TEM and a Bruker, Inc. Picoindenter with Nanoscale Electrical Contact Resistance (nanoECR). Experiments with electrical contacts utilized an electrical push-to-pull device (E-PTP) as shown in Figure 1 (a) [4]. Specific loading and observation directions were selected for the specimens, which were attached via focused ion beam (FIB) lift-out to the E-PTP device, as shown in Figure 1 (b-c). The specimens were deformed uniaxially in tension with the motivation to correlate dislocation behavior and applied current pulses. A sequence of tests was carried out by load control and with variable electrical current pulsing during the mechanical test. An example load function vs time is shown in Figure 1 (d).

After each test, videos of the dislocation behavior could be directly compared to the electrical and mechanical data. Our initial studies resulted in two types of dislocation motion. The first type of motions happened in less than $0.1 \mathrm{sec}$ where pre-existing dislocations escaped from the sample upon pulsing. The other type of motion was slower, with dislocations remaining in the sample for more than 1 second. In the latter case, dislocation motion could be observed through several consecutive steps.

Our observations found that during plastic deformation relatively large slip traces with dislocations nucleated from the edges could be triggered by the pulses, contributing to large displacement increases in the sample. Figure 2 shows an example of dislocation motion in Al where the motion could be correlated directly with the applied current that was turned on at $42 \mathrm{~s}$, as indicated by the arrow in Figure 1 (d). Examples such as this show that dislocation motion can be explained by small changes in the applied electrical current which enabled the dislocation to overcome barriers. In addition, dislocations tend to move with a faster speed and a longer distance during the pulsing period, compared to the purely mechanically-triggered movement. We will also discuss results such as these in the context of local joule heating which can be measured indirectly through expansion of the lattice measured using TEM diffraction. 
It is our ultimate goal to quantitatively measure the strain fields and local lattice change around dislocations to understand the external current effects in the plastic deformation of metals and alloys. Comparisons between the lattice strain fields, dislocation behavior and applied electric filed will provide valuable data to further improve atomistic models describing the dislocation motion change with electroplasticity. These experimental and theoretical frameworks will provide a scientific foundation for the design and optimization of alloys with enhanced electroformability for targeted manufacturing applications [5].

\section{References:}

[1] H Conrad et al., JOM 42.9 (1990), p. 28.

[2] WA Salandro et al., Springer 10.1007/978-3-319-08879-2 (2015).

[3] P Yiu, CH Hsueh and CH Shek, Journal of Alloys and Compounds 658 (2016), p.795.

[4] S Kaps, S Bhowmick and J Grottrup et al., ACS Omega 2 (2017), p. 2985.

[5] The authors gratefully acknowledge funding from the US Office of Naval Research under Grant No. N00014-17-1-2283. Work at the Molecular Foundry was supported by the Office of Science, Office of Basic Energy Sciences, of the U.S. Department of Energy under Contract No. DE-AC02-05CH11231.
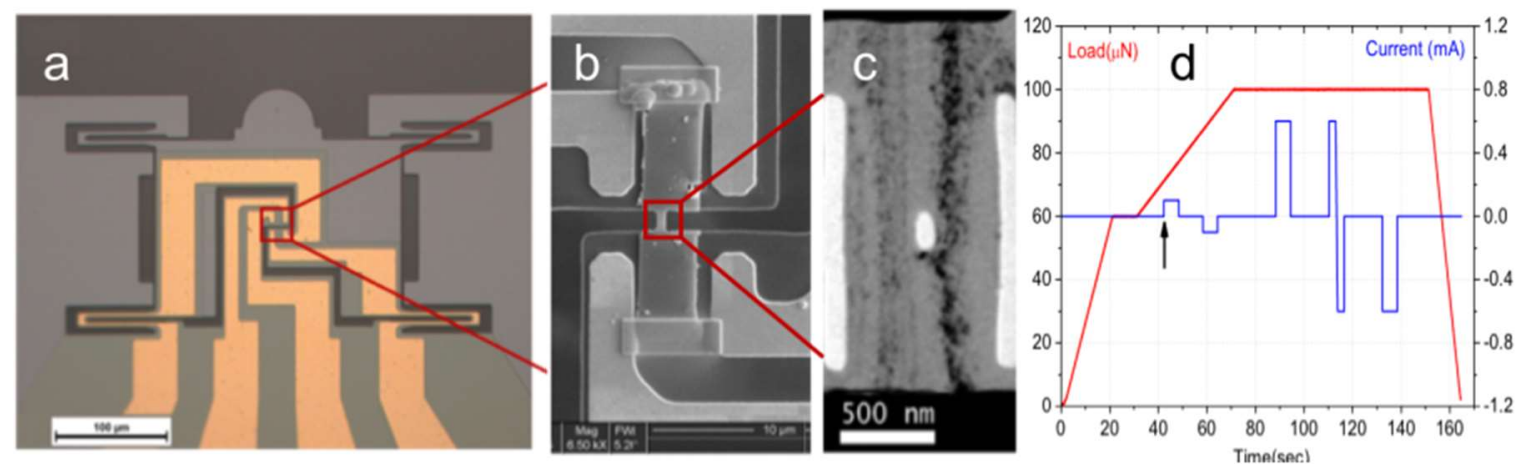

Figure 1. (a) The electric push-to-pull (E-PTP) device, (b) lift-out metal thin Al foil and (c) modified dog-bone shape with a hole for localization of stress for dislocation observation in TEM. (d) Load function vs time with current pulse applied during the tension test.

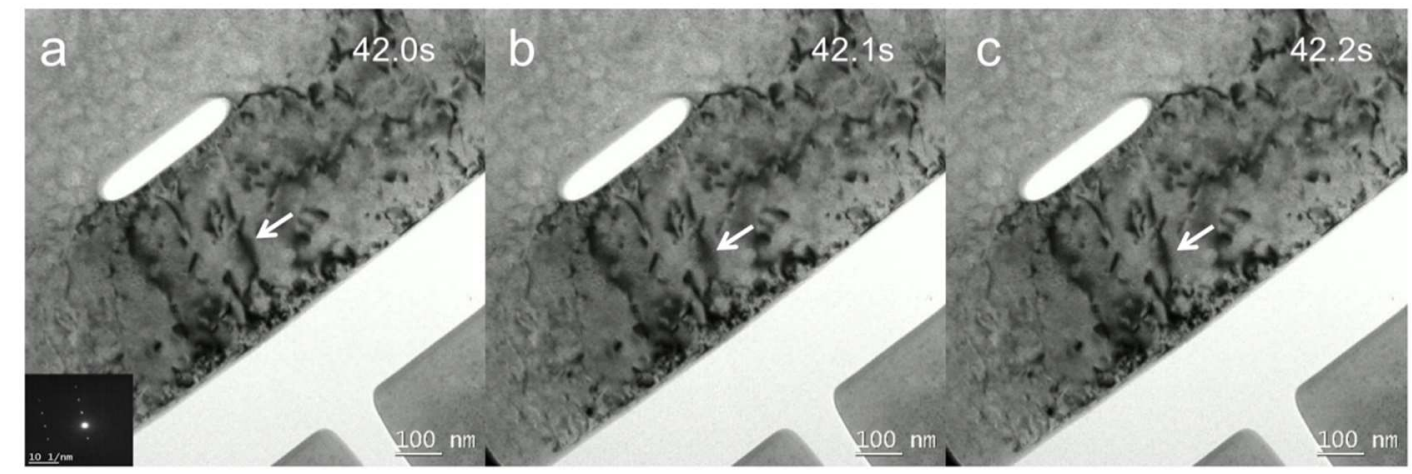

Figure 2. Example of dislocation movement in Al correlated with application of an applied electrical current at 42 second (corresponding to the black arrow in Figure 1d). The dislocation motion can be seen to occur within two frames. (a-c) Three consecutive images showing single dislocation depinning with the application of a current. 